\title{
Should the Pancreas be Routinely Included with an Intestinal Graft?
}

\author{
David F. Mercer ${ }^{1} \cdot$ Matthew J. Brady $^{1} \cdot$ Gaurav Jain $^{1}$
}

Published online: 8 April 2015

(C) Springer International Publishing AG 2015

\begin{abstract}
Intestinal transplant is the only curative solution for children and adults with life-threatening complications of intestinal failure. A dilemma that arises often in real life, typically at the time of organ allocation involving multiple centers, is whether the pancreas can and/or should be procured and transplanted separately from an intestine-containing graft. Evidence appears to support routinely allocating the pancreas along with a composite liver-intestine graft. For all pediatric recipients and recipients of any age requiring foregut replacement, the pancreas would likewise seem fundamental to the graft. The only scenario where the pancreas might not be considered as integral to the graft is an isolated intestine transplant where the recipient does not require the foregut. We feel that the pancreas should generally be allocated with isolated intestine grafts in adults, as the added benefit to the pancreas recipient pool is not adequately offset by the risk to the intestine recipients.
\end{abstract}

Keywords Intestinal transplantation $\cdot$ Multivisceral transplantation $\cdot$ Organ procurement $\cdot$ Pancreas transplantation

This article is part of the Topical Collection on Pancreas Transplantation

David F. Mercer

dmercer@unmc.edu

Matthew J. Brady

matthew.brady@unmc.edu

Gaurav Jain

gaurav.jain@unmc.edu

1 Section of Solid Organ Transplantation, Department of Surgery, University of Nebraska Medical Center, 983285 Nebraska Medical Center, Omaha, NE 68198-3285, USA

\section{Introduction}

Intestinal transplant is the only curative solution for children and adults with life-threatening complications of intestinal failure. Due to inadequate absorptive surface area, patients are unable to sustain their own nutrition and/or hydration by enteral means alone and become dependent on parenteral fluids and nutrients. While initially life saving, parenteral nutrition ultimately has hepatotoxic effects which can lead to irreversible liver disease and the need for transplant. The necessity for long-term central venous access can result in thrombosis of access sites or recurrent central line-associated blood stream infections, both accepted indications for transplant. Additional indications, slowly developed over time, include severe motility disorders, certain low-grade tumors (e.g., desmoid, neuroendocrine) involving the mesenteric root, diffuse splanchnic thrombosis, an unreconstructable GI tract, and in selected cases, poor quality of life on PN.

The small intestine may be transplanted as an isolated organ or as a part of a composite graft. With the small intestine as the focal point of the graft, additional transplanted organs have included pancreas, spleen, stomach, kidneys, right colon, and liver. This wide variety of options has led to little consistency and perhaps some confusion in the literature about nomenclature. For the majority of indications, other than the intestine and the liver, additional organs are not fundamental requirements for the recipient, and essentially equivalent results have been reported with and without the inclusion of each component [1-4]. For purposes of discussion in this review, two major graft types will be specified: intestine and liver intestine.

The presence or absence of the liver in a graft dictates the essential technical performance of the recipient operation and also to a significant extent the outcomes of both patient and graft survival $[5,6]$. Liver-containing grafts have venous 
outflow constructed similar to a liver transplant, either bicaval or piggyback, with arterial inflow from an aortic conduit or aortic patch; the graft portal vein is left untouched. In contrast, liver-exclusive grafts have their venous outflow constructed using the portal vein which may be drained either systemically to the vena cava or portally to the superior mesenteric vein (SMV)/portal vein complex; arterial inflow is typically direct to the graft superior mesenteric artery (SMA), or to an aortic patch containing that vessel.

A dilemma that comes up often in real life, typically at the time of organ allocation involving multiple centers, is whether the pancreas can and/or should be procured and transplanted separately from an intestine-containing graft. The center performing the intestinal transplant is often advocating for inclusion of the pancreas with the intestine graft, while a potential pancreas center advocates for procurement of the pancreas separately, to be used in a putative pancreas or kidney/ pancreas recipient. This has (not infrequently) led to cases where intestine recipients have been denied access to organs because of insistence on the pancreas being allocated separately and, conversely, to high-quality pancreata being lost to the pancreas recipient pool, when allocated "needlessly" to an intestine recipient. Allocation decisions are typically organ procurement organization (OPO)-specific and often reflect the bias of one or two dominant centers in that OPO. The essential question in this argument, and the purpose of this review, becomes is the pancreas itself fundamental to the intestine graft, in which case it should be included routinely with the intestine graft, or should it be allocated separately? To address this question, we reviewed the literature on the role of the pancreas in intestine-containing grafts, in an attempt to suggest rational solutions.

\section{Liver-Intestine Grafts}

In the original description of liver-intestine grafts in humans, Starzl performed the operation using an aortic conduit with the distal aorta of the graft (caudal to the origin of the inferior mesenteric artery) being anastomosed to the recipient infrarenal aorta, and piggybacked caval outflow [7]. The entire pancreaticoduodenal complex of the graft was left intact. During the early development of the field, inclusion of the pancreas with the composite graft was most typical and advocated because of the technical advantages it conferred by obviating the need for biliary reconstruction.

When Grant et al. published the first long-term success with combined liver-intestine surgery, the technique involved skeletonization of the portal vein by removal of the duodenum and pancreas [8]. This technique was reported in early series from both Nebraska and Pittsburgh [9, 10]. As early experience developed, it was recognized that removal of the pancreaticoduodenal complex lead to specific challenges, as taken from Sudan et al. [11]: "difficult backtable preparation, especially in pediatric donors. (2) significant morbidity from bile leaks after biliary anastomosis to a Roux-en-Y loop of donor jejunum; and (3) torsion on the portal vein axis, both during backtable preparation and, particularly, while sewing the graft in with volvulus leading to venous congestion and graft ischemia." The operative technique changed to routinely include the donor duodenum and pancreas in the liverintestine graft, which simplified the procedure and alleviated these concerns.

Fishbein et al. did present a manuscript describing simultaneous noncomposite transplantation of a liver and an intestine as two separate grafts taken from the same donor [12]. This allowed the pancreas to be transplanted into a separate donor and permitted the subsequent removal of the intestine graft in the event of a severe rejection, with preservation of the hepatic graft. While possible technically, this operation added significantly to the operative steps and vascular anastomoses required. Due to technical challenges, they also felt this procedure would not be appropriate for pediatric procedures. Subsequent publication does not reference this technique, perhaps suggesting it was not adopted routinely [13].

Keeping the pancreas and duodenum intact with the graft does not add significant risk to the composite graft, as outlined by Vianna, Yersiz, and Papachristou [14-16]. Isolated rejection of the pancreas in the absence of rejection of the intestine or liver is essentially nonexistent. It has the significant advantage of simplifying both the donor and the recipient operations, permitting either the retention or removal of the native foregut as dictated by the clinical scenario. Removing it from the graft and discarding it as performed in the earliest series would only add morbidity to the procedure without contributing a pancreas to the recipient pool. Further, given the relatively small numbers of combined liver and intestine transplant performed annually, it is difficult to make an argument that the pancreas should be removed from the composite graft, necessitating separate transplants of the liver and the intestine and enhancing recipient risk, in order to provide a few additional pancreata to the recipient pool. The evidence would support routinely allocating and transplanting the pancreas along with a composite liver-intestine graft; it should be considered fundamental to the graft itself.

\section{Intestine-Based Grafts Excluding the Liver}

When considering intestine grafts not including a liver, the case for always leaving the pancreas with the intestine graft is not as clear-cut as with liver-intestine grafts.

In the instance where foregut replacement is required, termed a "modified multivisceral" transplant by the Pittsburgh group [5], the recipient has a need for the pancreaticoduodenal complex. In this case, the requirement of the intestine recipient 
is every bit as valid as that of a potential pancreas recipient, and the issue is handled adequately by the current allocation system. Clearly, in these cases, the pancreas is fundamental to this graft.

The only scenario where the pancreas might not be considered as integral to the graft is the case of an isolated intestine transplant where the recipient does not require the foregut. In these cases, the pancreas and duodenum are typically removed on the backtable, skeletonizing the graft portal vein and SMA (Fig. 1). This is done to provide additional length to both the inflow and outflow vessels and to permit the use of a Carrel patch on the graft artery. In this case, a potentially transplantable pancreas is in effect "discarded" to facilitate the intestine recipient procedure.

The technique to procure the pancreas separately from the bowel and then transplant each into different recipients is established [17]. By dividing the graft SMA and SMV at the inferior margin of the pancreas, there is shortening of each vessel (Fig. 1). To gain length on the vessels for implantation, sacrifice of the first jejunal branches may be required, which at least in part compromises the intestine graft, although perhaps not sufficiently to reduce its utility. Alternatively, the use of arterial and/or venous extension grafts to facilitate implantation may be required. The practical reality is that where a liver and a pancreas are being transplanted already, the iliac grafts are generally spoken for and thus are not available to the intestine transplant. It is possible to find other arterial and venous extension grafts, however, and where an adequate length of appropriate vein and artery can be provided to the intestine transplant team, this might not be prohibitive.

Fridell et al. examined the outcomes of pancreas grafts from donors where an isolated intestine was procured from the same donor [18•]. This manuscript includes a nice description of practical approach to the dual procurement. It clearly demonstrates that the pancreas can be transplanted safely after such procurement, with survival results both nationally and from their own center that are equivalent to SRTR expected rates. The effect of separate allocation of the pancreas on the fates of the intestine grafts is not as clear, however. From the UNOS data in the manuscript, the outcome of the intestine grafts was not presented, to determine if there was a greater risk of graft loss from technical complications. Further, the center-specific data describes 34 dual procurements with a $100 \%$ survival at 3 months of the intestines transplanted but does not reveal if all procured organs were transplanted or if some were not usable. It is not clear if extension grafts were used. These data would suggest that when transplanted, at the authors' institution, the graft does not seem to show early losses from technical complications but does not permit further conclusions nationally nor the effect of dual procurement and allocation on ability to use intestinal grafts.

Most surgeons would agree that transplantation of pediatric isolated intestine grafts is not possible without the additional vessel length and arterial patch provided by inclusion of the pancreas with the graft; there is simply not adequate justification for significantly enhancing the risk of graft thrombosis to a pediatric recipient to add an isolated pediatric pancreas to the donor pool. This then leaves only the circumstance of an isolated intestine graft being performed in an adult wherein the fate of the pancreas is at issue.

Based on the technical feasibility of isolated intestine transplantation in adults without the inclusion of the pancreas in the graft [17], one might reasonably conclude that the pancreas is not fundamental to the intestine graft. The argument for or against including the pancreas with the intestine becomes then to a large extent philosophical in nature. Is the enhanced risk to an intestine recipient - even if the incremental risk is small, balanced by the distributive risk to the entire population of potential pancreas recipients of removing an organ from the donor pool?
Fig. 1 Techniques for procurement and implantation of isolated intestine grafts. Left panels show technique for standard procurement (upper) and implantation (lower) of isolated intestine grafts, where pancreas is divided and removed. Right panels show modified technique for preservation of both pancreas and intestine grafts, with placement of interposition grafts. Dashed lines show anastomosis. $S M A / S M V$ superior mesenteric artery/vein, $D 4$ fourth portion of duodenum
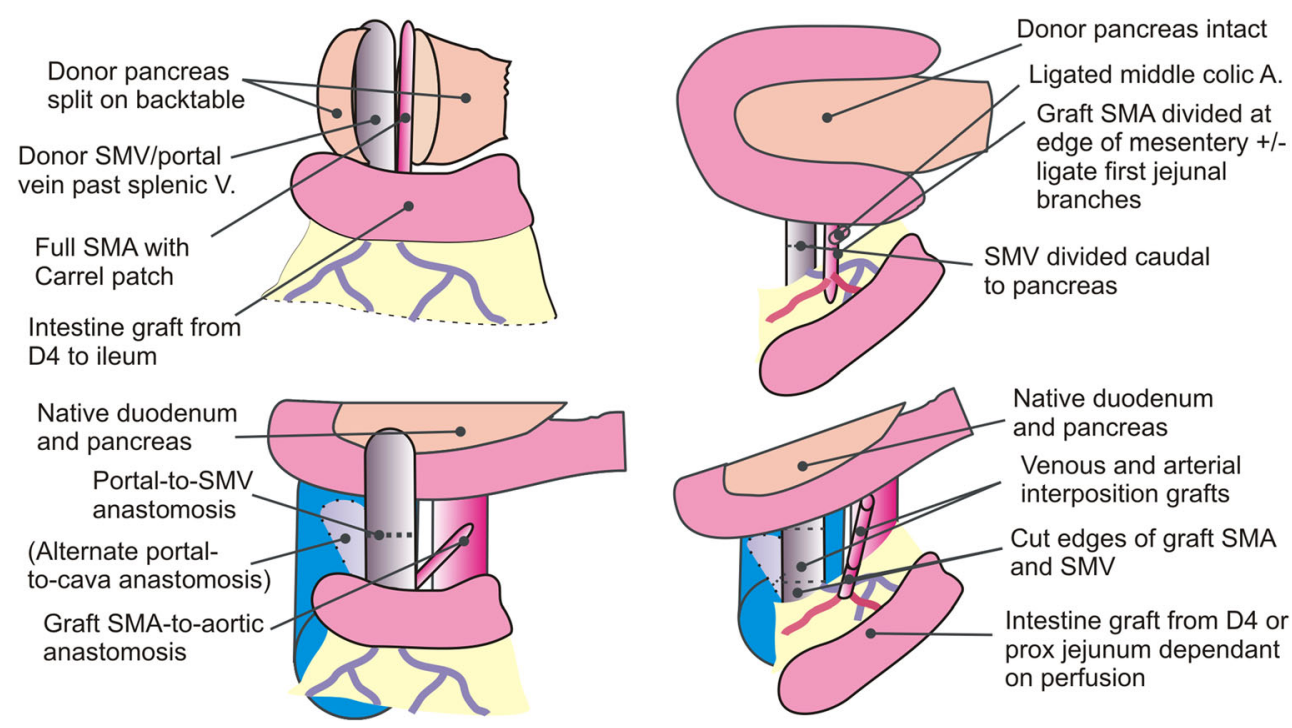
By combining the most recent Organ Procurement and Transplantation Network data (accessed January 15, 2015) and Intestinal Transplant Registry data, in the USA, there have been an average of about 72 adult intestine transplants performed yearly over the past 5 years, roughly half of which are isolated intestines [19]. This is compared with an average of 238 isolated pancreas transplants and 750 combined kidney/pancreas (KP) transplant, for a total of just under 1000 pancreata transplanted per year. If every adult isolated intestine graft was performed without the pancreas which was then transplanted separately, the total number of transplanted pancreata would increase each year by $\sim 4 \%$, and the waittime might be expected to reduce by about 24 days for isolated pancreas or KP recipients. This would come at the expense of increased donor operation complexity and enhanced risk to the intestine recipients due to the additional technical complexity. Given the small number of isolated intestine transplants performed each year, the loss of even one intestine graft from the pool from technical complication would, as a percentage, roughly offset the entire advantage accrued to the pancreas recipients from the additional organs.

At the present time, there is a separate organ acquisition cost for a pancreas in association with a multivisceral graft. In these cases, the pancreas is actually transplanted, and thus the cost would seem appropriate. In isolated small intestine transplants when the pancreas is not needed for the recipient but rather only for technical reasons, we suggest a separate acquisition cost is not appropriate, which is in line with the current system.

\section{Conclusion}

Based on published evidence, it would be correct to conclude that the pancreas is critical and fundamental to combined liver-intestine grafts, all foregut-containing intestine grafts, and pediatric isolated intestinal grafts. Loss of the pancreas from any of these grafts would unacceptably raise the risk to recipients. It would be our opinion that where possible, the pancreas should be allocated along with isolated intestine grafts in adults, as the added benefit to the pancreas recipient pool may not be adequately offset by the risk to the intestine recipients. Our opinion is clearly biased by our practice, however, and there is insufficient data making this the only acceptable argument. It may be of benefit to establish a consensus opinion to make allocation uniform across the country, although at present, it does not seem that either recipient population is dramatically harmed by the current system of ad hoc allocation decisions.

\section{Compliance with Ethics Guidelines}

Conflict of Interest David F. Mercer, Matthew J. Brady, and Gaurav Jain declare that they have no conflict of interest.

Human and Animal Rights and Informed Consent This article does not contain any studies with human or animal subjects performed by any of the authors.

\section{References}

Papers of particular interest, published recently, have been highlighted as:

- Of importance

1. Goulet O, Sauvat F, Ruemmele F, et al. Results of the Paris program: ten years of pediatric intestinal transplantation. Transplant Proc. 2005;37(4):1667-70.

2. Kato T, Selvaggi G, Gaynor JJ, et al. Inclusion of donor colon and ileocecal valve in intestinal transplantation. Transplantation. 2008;86(2):293-7.

3. Kato T, Tzakis AG, Selvaggi G, et al. Transplantation of the spleen: effect of splenic allograft in human multivisceral transplantation. Ann Surg. 2007;246(3):436-44. discussion 445-436.

4. Todo S, Reyes J, Furukawa H, et al. Outcome analysis of 71 clinical intestinal transplantations. Ann Surg. 1995;222(3):270-80. discussion 280-272.

5. Abu-Elmagd KM, Costa G, Bond GJ, et al. Five hundred intestinal and multivisceral transplantations at a single center: major advances with new challenges. Ann Surg. 2009;250(4):567-81.

6. Abu-Elmagd KM, Wu G, Costa G, et al. Preformed and de novo donor specific antibodies in visceral transplantation: long-term outcome with special reference to the liver. Am J Transplant. 2012;12(11):3047-60.

7. Starzl TE, Rowe MI, Todo S, et al. Transplantation of multiple abdominal viscera. JAMA. 1989;261(10):1449-57.

8. Grant D, Wall W, Mimeault R, et al. Successful small-bowel/liver transplantation. Lancet. 1990;335(8683):181-4.

9. Abu-Elmagd K, Todo S, Tzakis A, et al. Three years clinical experience with intestinal transplantation. J Am Coll Surg. 1994;179(4): 385-400.

10. Langnas AN, Shaw Jr BW, Antonson DL, et al. Preliminary experience with intestinal transplantation in infants and children. Pediatrics. 1996;97(4):443-8.

11. Sudan DL, Iyer KR, Deroover A, et al. A new technique for combined liver/small intestinal transplantation. Transplantation. 2001;72(11):1846-8.

12. Fishbein T, Florman S, Gondolesi G, Decker R. Noncomposite simultaneous liver and intestinal transplantation. Transplantation. 2003;75(4):564-5.

13. Matsumoto CS, Fishbein TM. Modified multivisceral transplantation with splenopancreatic preservation. Transplantation. 2007;83(2):234-6.

14. Papachristou GI, Abu-Elmagd KM, Bond G, et al. Pancreaticobiliary complications after composite visceral transplantation: incidence, risk, and management strategies. Gastrointest Endosc. 2011;73(6):1165-73. 
15. Vianna R, Fridell JA, Mangus R, Hollinger EF, Matiosky A, Tector AJ. Safe inclusion of the entire pancreas as a component of the multivisceral graft. Transplantation. 2008;86(1):114-6.

16. Yersiz H, Renz JF, Hisatake GM, et al. Multivisceral and isolated intestinal procurement techniques. Liver Transpl. 2003;9(8):881-6.

17. Abu-Elmagd K, Fung J, Bueno J, et al. Logistics and technique for procurement of intestinal, pancreatic, and hepatic grafts from the same donor. Ann Surg. 2000;232(5):680-7.
18. Fridell JA, Mangus RS, Powelson JA, Vianna RM, Tector AJ. Outcomes of pancreas allografts procured simultaneously with an isolated intestine allograft: single-center and national data. Transplantation. 2012;94(1):84-8. This manuscript shows technique for separation of pancreas and small intestine grafts and provides some contemporary data on outcomes.

19. Grant D, Abu-Elmagd K, Mazariegos G, et al. Intestinal transplant registry report: global activity and trends. Am J Transplant. 2015;15(1):210-9. 